\title{
Comparison of 3-D Synthetic Aperture Phased-Array Ultrasound Imaging and Parallel Beamforming
}

Rasmussen, Morten Fischer; Jensen, Jørgen Arendt

Published in:

IEEE Transactions on Ultrasonics, Ferroelectrics and Frequency Control

Link to article, DOI:

10.1109/TUFF c .2014 .006345

Publication date:

2014

Link back to DTU Orbit

Citation (APA):

Rasmussen, M. F., \& Jensen, J. A. (2014). Comparison of 3-D Synthetic Aperture Phased-Array Ultrasound Imaging and Parallel Beamforming. IEEE Transactions on Ultrasonics, Ferroelectrics and Frequency Control, 61(10), 1638-1650. https://doi.org/10.1109/TUFF c .2014.006345

\section{General rights}

Copyright and moral rights for the publications made accessible in the public portal are retained by the authors and/or other copyright owners and it is a condition of accessing publications that users recognise and abide by the legal requirements associated with these rights.

- Users may download and print one copy of any publication from the public portal for the purpose of private study or research.

- You may not further distribute the material or use it for any profit-making activity or commercial gain

- You may freely distribute the URL identifying the publication in the public portal 


\title{
Comparison of 3-D Synthetic Aperture Phased-Array Ultrasound Imaging and Parallel Beamforming
}

\author{
Morten Fischer Rasmussen and Jørgen Arendt Jensen, Fellow, IEEE
}

\begin{abstract}
This paper demonstrates that synthetic aperture imaging (SAI) can be used to achieve real-time 3-D ultrasound phased-array imaging. It investigates whether SAI increases the image quality compared with the parallel beamforming (PB) technique for real-time 3-D imaging. Data are obtained using both simulations and measurements with an ultrasound research scanner and a commercially available 3.5$\mathrm{MHz}$ 1024-element 2-D transducer array. To limit the probe cable thickness, 256 active elements are used in transmit and receive for both techniques. The two imaging techniques were designed for cardiac imaging, which requires sequences designed for imaging down to $15 \mathrm{~cm}$ of depth and a frame rate of at least $20 \mathrm{~Hz}$. The imaging quality of the two techniques is investigated through simulations as a function of depth and angle. SAI improved the full-width at half-maximum (FWHM) at low steering angles by $35 \%$, and the 20 -dB cystic resolution by up to $62 \%$. The FWHM of the measured line spread function (LSF) at $80 \mathrm{~mm}$ depth showed a difference of $20 \%$ in favor of SAI. SAI reduced the cyst radius at $60 \mathrm{~mm}$ depth by $39 \%$ in measurements. SAI improved the contrast-to-noise ratio measured on anechoic cysts embedded in a tissue-mimicking material by $29 \%$ at $70 \mathrm{~mm}$ depth. The estimated penetration depth on the same tissue-mimicking phantom shows that SAI increased the penetration by $24 \%$ compared with PB. Neither SAI nor PB achieved the design goal of $15 \mathrm{~cm}$ penetration depth. This is likely due to the limited transducer surface area and a low SNR of the experimental scanner used.
\end{abstract}

\section{INTRODUCTION}

Since the 1950s, the usability and quality of ultrasound Simaging has improved because of advancements made in integrated electronics, algorithms, computing power, and transducer technology [1]-[3]. This has led to an increase in resolution, contrast, and penetration depth. These technology advancements have led to high-quality 2-D and 3-D imaging systems. Some state-of-the-art 3-D imaging systems have more than 9000 active elements, beamformers integrated directly in the ultrasound probe, and some require water cooling. These ultrasound systems are therefore very expensive both to develop and to produce. The purpose of this paper is to investigate the achievable 3-D image quality possible with a modest number

Manuscript received February 13, 2014; accepted June 4, 2014. This research was financially supported by grant 024-2008-3 from the Danish Advanced Technology Foundation, by the Technical University of Denmark, and by BK Medical ApS, Denmark.

The authors are with the Center for Fast Ultrasound Imaging, Department of Electrical Engineering, Technical University of Denmark, Kgs. Lyngby, Denmark (e-mail: research@mofi.dk).

DOI http://dx.doi.org/10.1109/TUFFC.2014.006345 of active elements. It is investigated which beamforming technique of synthetic aperture imaging (SAI) [2], [4]-[7] and parallel beamforming (PB) [8], [9] that achieves the best image quality. Earlier work by the authors investigated the performance of 3-D synthetic aperture imaging and parallel beamforming using simulations [10] and phantom studies [11]. In this paper, a more realistic setup is used, using only 256 active elements and beamformer channels as opposed to 1024 active elements and beamformers. The measurements are also expanded, now including cysts and tissue-mimicking phantom measurements.

Two major obstacles have delayed the implementation of real-time 3-D imaging systems: the large number of channels on a 2-D array transducer to scan the volume and the low frame rate often achievable when scanning a full volume. Parallel beamforming addresses the frame rate problem by broadening the transmit beam and focusing multiple receive beams in parallel. This increases the frame rate in proportion to the number of lines beamformed per emission. The downside compared with emitting a focused beam for each receive-beamformed line is a lower resolution and an amplitude modulation artifact of the beamformed lines. Different measures have previously been pursued to minimize the parallel beamforming artifact [12]-[14], these are not investigated or applied in this work. Parallel beamforming was, in the form of Explososcan [9], [15] implemented in hardware by Smith, von Ramm, and colleagues as early as the 1980s.

Several versions of SAI exist [5], [7], [16], [17]. In this paper, SAI refers to synthetic transmit focusing, in which a virtual transmit aperture is synthesized [18]-[20]. Using synthetic transmit focusing, an entire volume can be dynamically focused in transmit, whereas conventional transmit focusing only has a fixed focal depth in transmit.

Related work was carried out by Karaman et al., who increased the SNR of synthetic aperture imaging by transmitting from multiple elements to emulate a single powerful virtual transmit element [18]. Achieving highframe-rate 3-D synthetic aperture imaging by mechanically sweeping a 1-D phased array was investigated by Lockwood et al. [21]. Nikolov and Jensen [22] used the elevation focal point as a virtual source in the elevation direction with a mechanically scanned 1-D transducer array. A thorough investigation of 2-D synthetic aperture phased-array imaging was made by Johnson et al. [23], [24]. They showed that for optimal image quality when the number of active elements is limited, the active subarray must be translated between the emissions. High-frame- 
rate 3-D imaging with a 2-D phased array was investigated via simulations by Kim et al. [25].

Synthetic aperture imaging is susceptible to tissue motion artifacts, which degrade the image quality. Previous literature has demonstrated tissue motion compensation in the axial direction [6], [26], [27] and in two dimensions [28]-[30]. Because the tissue inherently moves in 3-D, tissue motion compensation should ideally be carried out in all three dimensions when performing 3-D imaging. To our knowledge, reliable 3-D tissue motion compensation has yet to be demonstrated. 3-D tissue motion compensation is therefore not investigated nor applied in this work.

Tissue harmonic imaging is routinely applied when performing conventional clinical cardiac imaging. Synthetic aperture tissue harmonic imaging has been demonstrated by Bae et al. [31], who band-pass filtered the second harmonic before beamforming, and by Rasmussen et al. [32], who applied pulse inversion. Generating the second harmonics requires sufficient acoustic pressure. This demonstrates that tissue harmonic imaging can be performed with both parallel beamforming and synthetic aperture imaging. In this paper, the image quality is investigated for the fundamental harmonic.

In the next section, the two techniques are further introduced and the imaging sequences are designed and optimized. In Section III, the imaging quality is investigated by simulations of the point spread function (PSF) at a large number of positions within the imaged volume. The PSF simulations show that synthetic aperture imaging improves the detail resolution at small steering angles, compared with parallel beamforming. With larger steering angles, the detail resolution performance of the two techniques is similar. The contrast resolution is significantly improved by synthetic aperture imaging at all positions in the entire imaged volume. In Section IV, the imaging quality is investigated using wire and cyst phantom measurements. The wire phantom measurements are carried out at a low steering angle and show the same tendency as the simulations; synthetic aperture imaging increases resolution as well as contrast. The cyst phantom measurement confirms that synthetic aperture imaging increases the contrast at all depths, compared with parallel beamforming. Measurements on a tissue-mimicking phantom indicate that the penetration depth is deeper for synthetic aperture imaging compared with parallel beamforming. Discussion and the conclusion follow in Section V.

\section{Methods}

In this section, the implementations of the two techniques are described. Certain restrictions must be imposed for the comparison to be realistic, such as the width of the aperture, the number of active channels used, and the use of multiplexing. First, these restrictions are determined, then an image quality metric, cystic resolution, is introduced, and finally, implementation and optimization of both techniques are carried out using simulations.
The two imaging techniques are designed for cardiac imaging, which requires imaging down to $15 \mathrm{~cm}$ and a frame rate $f_{\mathrm{r}}$ of at least $20 \mathrm{~Hz}$. To be comparable with products from the medical ultrasound industry, a volume scan spanning $90^{\circ}$ in both the azimuth and elevation direction is chosen.

With a maximum scan depth $r_{\max }$ of $15 \mathrm{~cm}$ and a speed of sound $c$ equal to approximately $1540 \mathrm{~m} / \mathrm{s}$, the maximum pulse repetition frequency is

$$
f_{\text {prf }}=\frac{c}{2 r_{\max }}=5.133 \mathrm{kHz}
$$

The possible number of emissions per frame then becomes

$$
N_{\mathrm{ems}}=\frac{5.133 \mathrm{kHz}}{20 \mathrm{~Hz}} \approx 256 .
$$

Using 256 emissions per frame allows for resolution of the azimuth and elevation directions with $\sqrt{256}=16$ emissions each.

Because of the constant increase in processing power of ultrasound scanners, the number of ultrasound channels that can be processed increases with time. A limiting factor is the diameter and weight of the cable connecting the ultrasound probe with the ultrasound system. If the cable is too heavy, the operator will risk injuring his or her wrist when scanning on a daily basis. To impose a limit on the cable size, both techniques are therefore limited to 256 active channels at a time during both transmitting and receiving. Multiplexing electronics in the handle that selects the active transducer elements are assumed to be available, and are otherwise neglected in this study.

\section{A. An Image Quality Metric: Cystic Resolution}

Two quality metrics are used for evaluating the images. The full-width at half-maximum (FWHM) and the cystic resolution. The cystic resolution describes the ability to detect an anechoic cyst in a uniform scattering medium [33]. Specifically, the cystic resolution gives the intensity at the center of an anechoic cyst of a given size relative to its surrounding uniformly back-scattering medium. The lower the intensity at the center of the cyst, i.e., the darker it appears in the image for a given cyst size, the better the imaging performance of the system. This metric mimics an often seen situation in medical ultrasound in which the object of interest is a weakly reflecting medium, such as blood or a cyst, surrounded by a stronger back-scattering medium. The relative intensity (RI) of the anechoic cyst was shown by Ranganathan and Walker [34], to be quantized as the clutter-energy-to-total-energy ratio,

$$
\operatorname{RI}(R)=\sqrt{\frac{E_{\text {out }}(R)}{E_{\text {tot }}}}=\sqrt{1-\frac{E_{\text {in }}(R)}{E_{\text {tot }}}},
$$

where $E_{\text {in }}$ is the signal energy inside a circular region with radius $R$ centered at the peak of the point spread function, 
$E_{\text {tot }}$ is the total point spread function energy, and $E_{\text {out }}$ is the point spread function energy outside the circular region. To achieve a single number from the $\mathrm{RI}(R)$-curve, one either determines the relative intensity for a single cyst radius, or determines the required radius to achieve a certain relative intensity, e.g., the cyst radius at which the intensity at center of the cyst is $20 \mathrm{~dB}$ lower than its surroundings, written as $R_{20 \mathrm{~dB}}$. In this work, the cystic resolution is presented as the cyst radius $R_{20 \mathrm{~dB}}$.

\section{B. Parallel Beamforming}

For 3-D imaging, parallel beamforming makes $N \times N$ receive lines per emission. From trial and error, $N=4$ scan lines per dimension were determined as an adequate spatial sampling frequency to represent the PSF at the focal point.

As determined in the previous section, the maximum $f_{\text {prf }}$ allows for 16 emissions per steering angle, leading to 16 $\times 4=64$ scan lines to be beamformed per dimension per emission. Beamforming $4 \times 4$ lines in parallel per emission gives a frame rate increase of 16 times compared with conventional phased-array imaging.

The transmit beam should therefore be four times as wide as the receive beams. In Fig. 1, a transducer array which focuses its transmit beam at the marked focal point is shown. The transmit beam width in radians is $\alpha$, the aperture width is $w$ and the focal depth $r$. Ideally, the transmit beam should have a main-lobe width of $\alpha=$ $90^{\circ} / 16=5.63^{\circ}$. As seen in Fig. 1, when the focus point is directly under the center of the transducer, a right-angled triangle can be drawn from the three points: the center of the active aperture, the focal point and the half-width at half-maximum (HWHM) of the transmit beam at the focus depth. Using this triangle and HWHM $=\mathrm{FWHM} / 2$ $\approx(\mathrm{f}$-number $\times \lambda) / 2$, the following approximate relation can be written

$$
2 \tan \frac{\alpha}{2}=\frac{\text { HWHM }}{r} \approx \frac{\text { f-number } \times \lambda}{2 r}=\frac{\lambda}{2 w}
$$

$\Uparrow$

$$
w \approx \frac{\lambda}{2 \tan \frac{\alpha}{2}}
$$

where $\lambda$ is the wavelength of the emitted wave and the f-number $=r / w$. In (4), $\tan (\alpha / 2)$ is assumed nonzero, implying that the main-lobe width is always nonzero. Inserting the values for $\lambda$ and $\alpha$ into (4b) gives an aperture width of $w=5.22 \mathrm{~mm}$. Table I shows the parameters of the $32 \times 32$ element 2-D transducer array used for both simulations and measurements. The pitch is $300 \mu \mathrm{m}$ in both directions. Therefore, an aperture width of $5.22 \mathrm{~mm}$ corresponds approximately to a 17-element-wide aperture. A circular aperture with a diameter of 17 elements contains approximately 226 elements, however 256 active channels are available for the scanner in both transmit and receive.

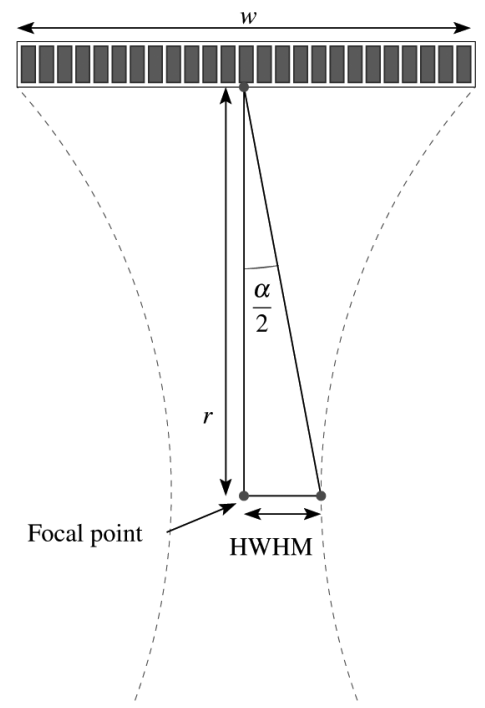

Fig. 1. Geometry for determining aperture width $w$, which approximately relates to a given beam width $\alpha . r$ is the distance from the transducer surface to the focal point.

If the diameter of the circular aperture is increased to 18 elements, it contains approximately 256 elements. The circular aperture with 256 active elements is shown in Fig. 2 (a). Because the transducer array is made of four stacked piezocrystals, the array contains three joints where no elements are present.

It has been shown [35], [36] that when the number of active channels is restricted and a wide aperture is needed, the simple sparse cross array results in one of the best ratios between imaging quality and the number of active elements used. Three possible transmit cross array apertures of differing widths are shown in Figs. 2(b)-2(d).

Because all shown transmit apertures in Fig. 2 are wider than $w$ from (4b), their transmit beam width will be too narrow. To compensate for this, the effective width of the apertures is reduced by applying an apodization function. The Tukey apodization function has a parameter $\psi$ that changes the apodization continuously from a rectangular apodization $(\psi=0)$ to a Hann apodization $(\psi=1)$. The $\psi$ parameter of the Tukey apodization, which leads to the correct width, is determined by numerical optimization. The optimization routine determines the $\psi$ parameter, which results in an area of the Tukey apodization function equal to the area of the rectangular apodization function. The optimized expression is thereby

$$
\underset{\psi}{\arg \min } f(\psi)=\left(\sum_{n=1}^{N} \operatorname{Tukey}(n ; \psi)-\sum_{m=1}^{M} \operatorname{rect}(m)\right)^{2} .
$$

Four different transmit apertures are shown in Figs. 2(a) $-2(d)$. They are optimized using (5) to have equal main-lobe size. The radiation pattern at the focal point and in the far field can be approximated by the Fourier transform of the active aperture [37, p.12], [38]. The 1-D Fourier transform of the center row of each transmit array is calculated and plotted in Fig. 2(e). The Fourier trans- 
Table I. Transducer, Simulation, and Measurement Parameters.

\begin{tabular}{|c|c|c|c|}
\hline Parameter name & Notation & Value & Unit \\
\hline \multicolumn{4}{|l|}{ General parameters } \\
\hline Frame rate & $f_{\mathrm{r}}$ & 20 & $\mathrm{~Hz}$ \\
\hline Pulse repetition frequency & $f_{\text {prf }}$ & 5.133 & $\mathrm{kHz}$ \\
\hline Emissions per frame & $N_{\text {ems }}$ & 256 & - \\
\hline Number of active elements & $N_{\text {act }}$ & 256 & - \\
\hline Scan depth (max range) & $r_{\max }$ & 15 & $\mathrm{~cm}$ \\
\hline Emission center frequency & $f_{0}$ & 3.0 & $\mathrm{MHz}$ \\
\hline Emission cycles & $N_{\text {cyc }}$ & 4 & - \\
\hline Emission window & $W_{\mathrm{ems}}$ & Hann & - \\
\hline \multicolumn{4}{|l|}{ Ultrasound probe parameters } \\
\hline Center frequency & $f_{\mathrm{c}}$ & 3.5 & $\mathrm{MHz}$ \\
\hline Relative bandwidth & BW & 66 & $\%$ \\
\hline Transducer pitch $-x$ & $d_{x}$ & 300 & $\mu \mathrm{m}$ \\
\hline Transducer pitch $-y$ & $d_{y}$ & 300 & $\mu \mathrm{m}$ \\
\hline Number of elements $-x$ & $N_{x}$ & 32 & - \\
\hline Number of elements $-y$ & $N_{y}$ & 35 & - \\
\hline Number of wired elements & $N_{\text {tot }}^{9}$ & 1024 & - \\
\hline Inactive rows & - & $9,18,27$ & - \\
\hline \multicolumn{4}{|l|}{ Simulation parameters } \\
\hline Sampling frequency & $f_{\text {S_sim }}$ & 120 & $\mathrm{MHz}$ \\
\hline Speed of sound & $c_{\mathrm{sim}}$ & 1540 & $\mathrm{~m} / \mathrm{s}$ \\
\hline \multicolumn{4}{|l|}{ Measurement parameters } \\
\hline Sampling frequency & $f_{\mathrm{s} \_ \text {meas }}$ & 70 & $\mathrm{MHz}$ \\
\hline $\mathrm{TX}$ voltage & $V_{\mathrm{TX}}$ & \pm 100 & $\mathrm{~V}$ \\
\hline Sound speed in water & $c_{\mathrm{w}}$ & 1482 & $\mathrm{~m} / \mathrm{s}$ \\
\hline Sound speed in phantom & $c_{\mathrm{pht}}$ & 1548 & $\mathrm{~m} / \mathrm{s}$ \\
\hline Attenuation in phantom & $\alpha$ & 0.51 & $\mathrm{~dB} /(\mathrm{MHz} \cdot \mathrm{cm})$ \\
\hline Wavelength in water & $\lambda_{\mathrm{w}}$ & 494 & $\mu \mathrm{m}$ \\
\hline Wavelength in phantom & $\lambda_{\text {pht }}$ & 516 & $\mu \mathrm{m}$ \\
\hline
\end{tabular}

form of the 17-element-wide rectangular array is plotted as a reference.

The main lobes of the four transmit apertures are seen to be practically identical, which shows the applicability of (5). The side lobes are seen to decrease faster for the wider apertures, e.g., apertures (c) and (d), compared with the narrower apertures, e.g., apertures (a) and (b). On the other hand, the wider the aperture is and the more the aperture is apodized, the less energy is emitted. As a trade-off between emitted energy and side-lobe performance, the 24-element-wide cross array, seen in Fig. 2(b), is chosen as the transmit aperture.

To get a wide receive aperture and thereby a narrow receive beam main-lobe, the cross array is also used in receive. In receive, 64 lines are beamformed per dimension, giving a main-lobe width of $\alpha_{\mathrm{RX}}=90^{\circ} / 64=1.41^{\circ}$. Inserting $\alpha_{\mathrm{RX}}$ in (4b) gives an aperture width of $17.9 \mathrm{~mm}$ or approximately 60 elements. Because the available ultrasound probe is $32 \times 0.3=9.6 \mathrm{~mm}$ wide in each dimension, and $32 \times 0.33 \mathrm{~mm} \times \sqrt{2}=13.6 \mathrm{~mm}$ along the diagonals, this receive aperture cannot be implemented. The widest possible array, a cross array along the diagonals, is chosen as receive aperture. Because the receive aperture is too narrow, it is apodized with a Tukey function with a $\psi$ parameter close to zero. The receive aperture is shown in Fig. 3.

The last parameter to be determined for the parallel beamforming technique is its focal depth. The influence of the focal depth on the imaging quality is investigated via simulations of the PSF using the ultrasound simulation program Field II [39], [40]. The PSF is investigated at a $0^{\circ}$ beam steering angle (straight down) at depths of 60,90 , 120 , and $150 \mathrm{~mm}$, while the transmit focal depth is varied from $5 \mathrm{~mm}$ to $120 \mathrm{~mm}$. The result of the parameter study is shown in Fig. 4. The main-lobe width in Fig. 4(a) is almost independent of the focal depth; it only improves locally in the region around the focal depth. In Fig. 4(b), the cystic resolution $R_{20 \mathrm{~dB}}$ is shown to be highly dependent on the focal depth, and it obtains a global minimum close to $30 \mathrm{~mm}$ of depth. The transmit focal depth of parallel beamforming is therefore chosen to be $30 \mathrm{~mm}$.

This optimized parallel beamforming setup is used for the rest of this paper.

\section{Synthetic Aperture Imaging}

To avoid grating lobes within the $\pm 45^{\circ}$ beamformed volume, the pitch of the transducer array should not be larger than $\lambda / 2$. The center frequency of the transducer array is $3.5 \mathrm{MHz}$ and the pitch is $300 \mu \mathrm{m}$, which corresponds to $0.68 \lambda$. As a compromise between the transducer efficiency in converting electrical to mechanical energy and grating-lobe levels, the center frequency is set to $3.0 \mathrm{MHz}$.

Synthetic aperture imaging synthesizes a virtual transmit aperture. One element is synthesized in the synthetic aperture for each focus point. Because 256 emissions are used, 256 virtual elements are synthesized. The relations between aperture array design and the PSF also apply to 


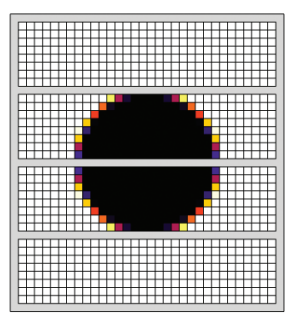

(a)

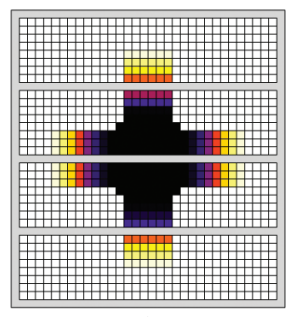

(c)

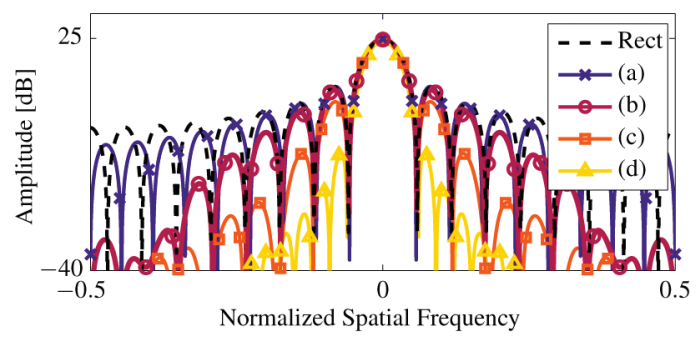

(e)

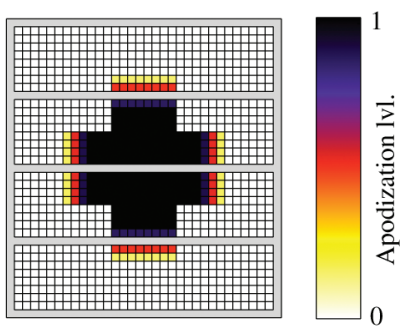

(b)

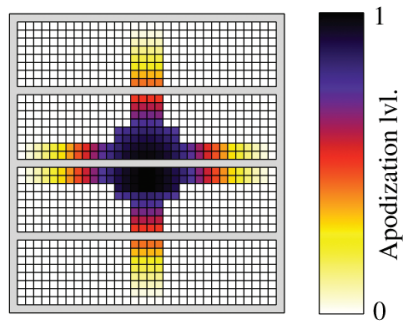

(d)
Fig. 2. (a)-(d) Four different transmit apertures with 256 active elements and approximately the same main-lobe width. (e) The 1-D Fourier transform of each of the four transmit apertures. The transmit aperture shown in (b) is chosen as the compromise between emitted energy and low side-lobe levels. The transmit aperture is used for all 256 emissions it takes to sample the volume.

the synthesized aperture array [19]. Especially, the width of the synthesized array and the pitch of the virtual elements must be considered. The location of the virtual elements also influences the distribution of the emitted energy, and thereby the SNR within the imaged volume.

The synthetic aperture can be synthesized in three different ways: by having a static transmit aperture and

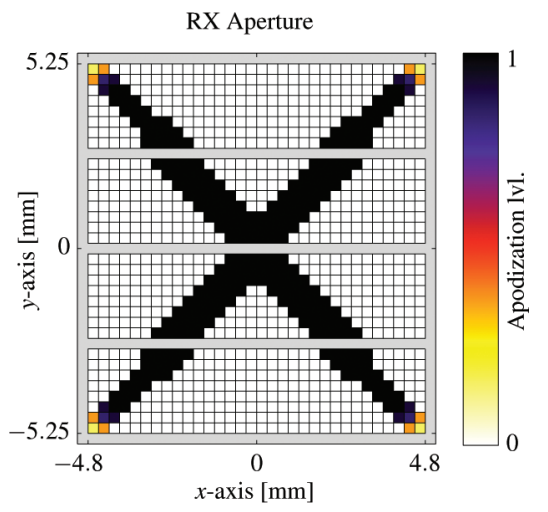

Fig. 3. The receive aperture for parallel beamforming is the widest possible cross array implementable on the $32 \times 32$ element array. It contains 256 active elements and is used for all 256 emissions.

steering the transmit beam angle for each emission, by translating transmit aperture and not applying beam steering, or by both translating the transmit aperture and applying beam steering. If a static transmit aperture with beam steering is used, the focal distance must be large, either in front of or behind the array, for the width of the synthesized aperture to become large enough. This leads to a large f-number, and, thus, a narrow beam. A narrow beam implies only little overlap between each transmit beam; thereby, only a small aperture is synthesized for any given point in the imaged volume. Usually, this leads to an inferior image quality. If the active aperture is translated and no beam steering is applied, the width of the synthesized array is independent of the transmit f-number. In this case, the synthesized array is narrower than the physical array. When no beam steering is applied, the energy of all transmit beams are emitted straight down. For phased-array imaging, this leads to the SNR being higher in front of the array, compared with the sides of the imaged volume. The third possibility is to both translate the physical array and to apply transmit beam steering. This has the advantage that even when the transmit f-number is kept low, a wide aperture can be synthesized. It is therefore chosen to both translate the active aperture and to apply beam steering of the transmit beam.

To enable the translation of the active aperture, it must be small to fit on the physical array and still leave room

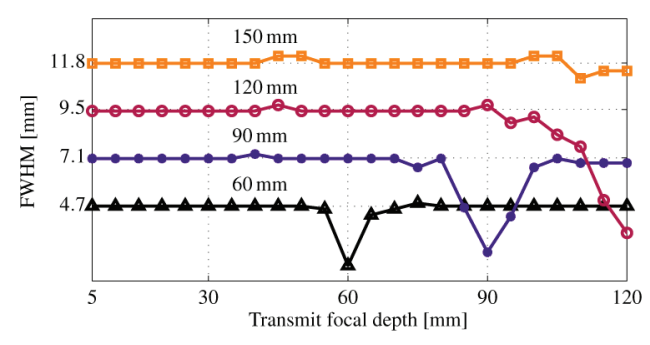

(a)

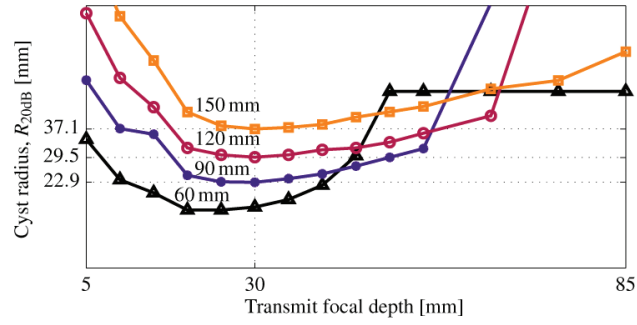

(b)

Fig. 4. Imaging quality metrics extracted from simulated PSFs. (a) The main-lobe width is shown to be almost independent of the transmit focal depth. (b) The cystic resolution $R_{20 \mathrm{db}}$ is shown to be highly dependent on the transmit focal depth, and it obtains a near global minimum at 30 mm of depth. 
TX Aperture

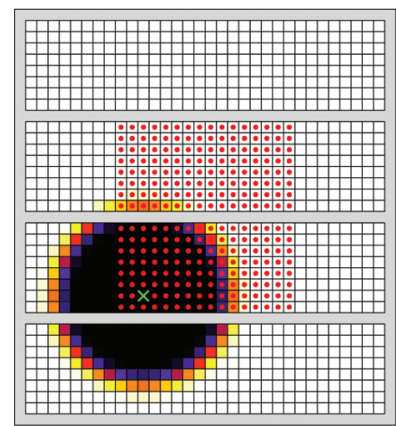

(a)
RX Aperture

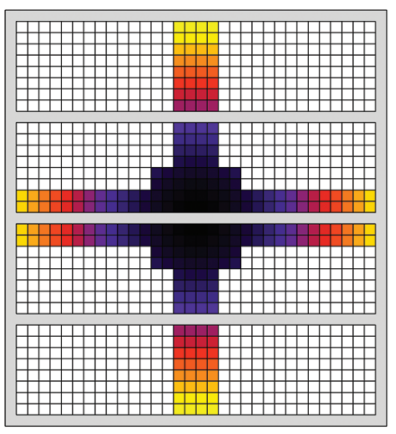

(b)
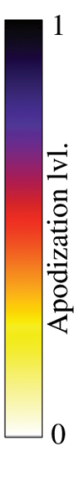

Fig. 5. The synthetic aperture imaging transmit and receive apodization implemented on the $32 \times 32$ element array. The transmit aperture translates between each emission. The center of the shown aperture is illustrated with a cross and the centers for the 255 remaining emissions are shown with a dot. The receive aperture is static during all 256 emissions.

for translation. The active array could either be a $16 \times 16$ square or a circle with radius of approximately 9 elements. To increase the circular symmetry of the PSF, the circular shape is chosen as active aperture.

A synthesized wide cross array would result in a small PSF but also in an unequal SNR performance with respect to steering angle. To assure an even SNR performance in all directions, within the pyramidal volume, the active aperture is translated around a square aperture with a side length of 16 elements. The centers of all translated transmit apertures are shown with a dot in Fig. 5(a). The cross is the center of the shown active aperture. The beam steering is controlled proportionally to the position of the active aperture. That is,

$$
\begin{aligned}
& \phi(x)=\kappa_{x} x \\
& \theta(y)=\kappa_{y} y
\end{aligned}
$$

where $\phi$ is the angle in the $z-x$ plane, $\theta$ is the angle in the $z$ - $y$ plane, $x$ and $y$ are the $(x, y)$ coordinates of the center of the active aperture, and $\kappa_{x}$ and $\kappa_{y}$ are scaling constants. $\kappa_{x}$ and $\kappa_{y}$ set the maximum transmit beam angle.

The focal depth of the transmit beam and the maximum transmit beam steering angle is determined by a parameter study using Field II. The detail resolution and cystic resolution are investigated at $60 \mathrm{~mm}$ depth at angles of $(\phi, \theta)=\left(0^{\circ}, 0^{\circ}\right)$ and $(\phi, \theta)=\left(30^{\circ}, 30^{\circ}\right)$. The result of the parameter study is shown in Fig. 6. If the maximum transmit beam steering is low, then the image quality is good in the center and poor at $30^{\circ}$. For large transmit beam steering angles and large focal depths, it is the opposite result. As a compromise, the focal depth is chosen to be $-6 \mathrm{~mm}$ and the maximum beam steering angle to $30^{\circ}$. The negative focal depth means that the focal point is located behind the transducer.

The resulting synthesized aperture is shown in Fig. 7. The transmit beam for the shown emission is illustrat-
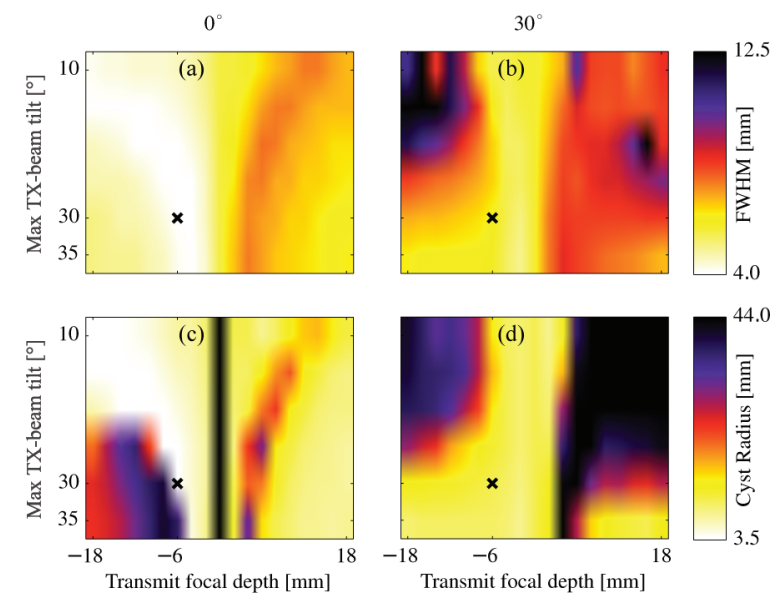

Fig. 6. Optimizing the transmit focal depth and the max transmit-beam angle of synthetic aperture imaging. In (a) and (c), the scatterer is located at an azimuth and elevation angle of $(\phi, \theta)=\left(0^{\circ}, 0^{\circ}\right)$; in (b) and (d), the azimuth and elevation angle is $(\phi, \theta)=\left(30^{\circ}, 30^{\circ}\right)$. In both cases, the depth of the scatterer is $60 \mathrm{~mm}$. The chosen parameter combination of a maximum transmit beam angle of $30^{\circ}$ and a focal depth of $-6 \mathrm{~mm}$ is marked with a cross.

ed with an arrow. The source of the beam is the active virtual source, shown with a circle. For each emission, a low-resolution volume is beamformed. Each point in the low-resolution volume is then weighted by a virtual source apodization. After the weighting, the low-resolution volumes are summed to create the high-resolution volume. The virtual source apodization has the shape of a cone centered around the transmit beam and with its apex located at the active virtual source. The angular width of the cone depends on the focal distance of the active transducer array. The closer the virtual source is to the transducer surface, the wider is the cone. In this work, the cone angular width is $50^{\circ}$. The beamformed points located outside of the cone are weighted by 0 . The points inside of the cone are weighted by a window function. In this work, a Hann-window is applied. The weight of a point within the cone depends on its angular distance to the transmit beam. This procedure removes the noise from the beamformed points which were not insonified by the transmit beam. This optimized setup of synthetic aperture imaging is used for the remainder of this paper.

\section{Simulations}

In this section, the image quality is investigated for the two techniques via simulations. The PSF at different spatial positions, as a function of depth and angle, are simulated.

\section{A. Simulation Setup}

The ultrasound simulation program Field II is used for the simulation study. A 2-D phased-array ultrasound probe, made by Vermon S.A. (Tours, France) with param- 


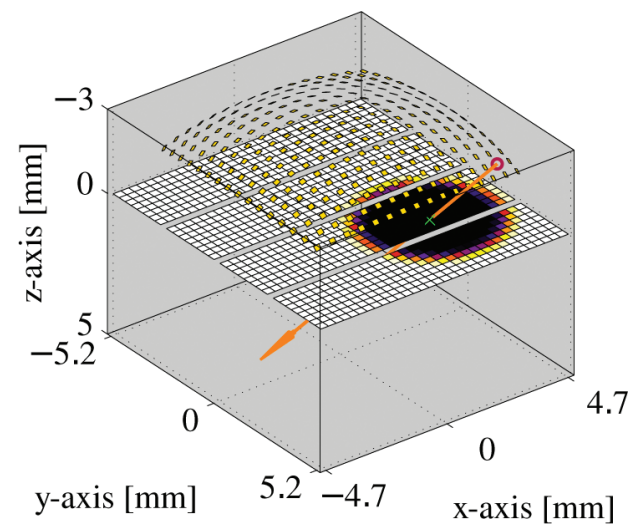

Fig. 7. The setup for a single emission is shown, which synthesizes one virtual element (shown as a circle) in the synthetic aperture. The remaining virtual elements of the sequence are shown as squares raised above the physical aperture. The virtual sources are located behind the aperture and the sound is emitted downwards, in the direction of the arrow. The cross marks the center of the active aperture and the colors of the physical elements represent their apodization value.

eters as shown in Table I, is emulated. The 2-D transducer has $35 \times 32$ elements, of which rows 9, 18, and 27 are inactive, effectively giving $32 \times 32$ individually controllable elements.

A single scatter is placed in front of the transducer array and then imaged using the optimized imaging sequences described in the previous section. The simulated receive signals are Hilbert-transformed, and the analytic samples are beamformed. After the Hilbert transform, the samples contain both the in-phase and the quadrature components of the signal and are now referred to as IQsamples or the IQ data set. The image quality metrics are applied on a 2-D slice of the 3-D PSF. The PSF in the axial direction is mostly dependent on the emission pulse, whereas in the $\phi$ - and $\theta$-directions it is mostly dependent on the imaging method. The PSFs are therefore sliced into a C-scan, a 2-D plane the shape of a spherical surface with a constant distance to the center of the transducer array. The two dimensions in the $\mathrm{C}$-scan are the two angles $\phi$ and $\theta$, which are also termed the azimuth and elevation angles, respectively.

\section{B. Simulation Results}

In Fig. 8, the PSF C-scan of both parallel beamforming and synthetic aperture imaging at a depth of $60 \mathrm{~mm}$ are shown as grayscale images with a dynamic range of $60 \mathrm{~dB}$. The main lobes of the two techniques are seen to be similar in both the elevation and azimuth directions. The sidelobe levels are clearly lower for synthetic aperture imaging than for parallel beamforming. The side lobes are seen to be asymmetrical, as they are wider in the elevation direction than in the azimuth direction. The asymmetric PSF is due to the asymmetry of the transducer array used. The transducer array contains three inactive rows of elements. These discontinuities cause the increased side-lobe levels in the elevation direction. Low-intensity grating lobes are

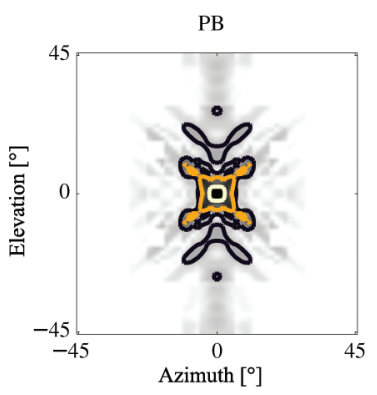

(a)

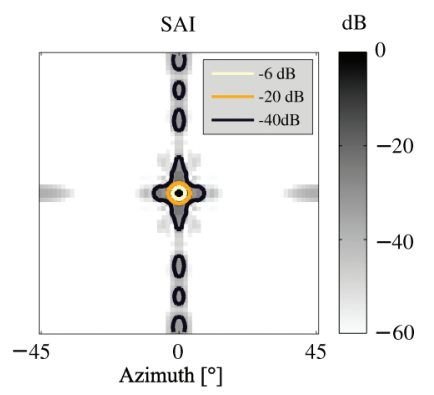

(b)
Fig. 8. Simulated C-scans of the 3-D PSFs of parallel beamforming and synthetic aperture imaging at a depth of $60 \mathrm{~mm}$. The dynamic range of the gray scale is $60 \mathrm{~dB}$. The main-lobe width is seen to be similar for the two techniques. The side lobes, especially at $-20 \mathrm{~dB}$, are lower for synthetic aperture imaging. The $-20-\mathrm{dB}$ isocurve in (a) is seen to have four distinct corners.

visible in the azimuth direction of the synthetic aperture imaging PSF. This is probably due to the pitch of both the physical and synthesized arrays being larger than $\lambda / 2$. In the elevation direction, it is hard to separate the side lobes from grating lobes.

The FWHM of the simulated PSFs in Fig. 9(a) is seen to be slightly better for synthetic aperture imaging than for parallel beamforming, at all depths. The difference between the two techniques increases with depth. The FWHM increases linearly with depth, which is expected for a phased-array system with a constant active aperture width. Parallel beamforming has a slightly increased FWHM performance at its transmit focal depth at $30 \mathrm{~mm}$, which is not expected. In the parallel beamforming PSF at $60 \mathrm{~mm}$ depth, shown in Fig. 8(a), the -20 -dB isocurve is seen to have four distinct corners. The intensity of the PSF at the four corners increases abruptly around $30 \mathrm{~mm}$ depth to above $-6-\mathrm{dB}$ intensity. This directly influences the FWHM measure and is the reason for the FWHM peak in Fig. 9(a). Because synthetic aperture imaging is transmit-focused at all depths, it has no specific depth with an unusually increased FWHM performance. Fig. 9(b) shows the FWHM at a depth of $100 \mathrm{~mm}$ as a function of steering angle. The FWHM increases slightly with increasing steering angle. This is also as expected, because the effective aperture width decreases with increasing steering angle. Parallel beamforming is seen to have a slightly better FWHM at increasing angles compared with synthetic aperture imaging.

In Fig. 10(a), the calculated cystic resolution described in Section II-A is plotted as a function of depth. The cystic resolution is seen to be significantly better for synthetic aperture imaging than for parallel beamforming. The difference between the two techniques increases with depth. The cystic resolution as a function of beam steering angle is plotted in Fig. 10(b). The difference in cystic resolution performance is largest when no beam steering angle is applied and decreases with increasing angle. It is also seen that the imaging performance of parallel beamforming oscillates once for each emission. The angles of the 


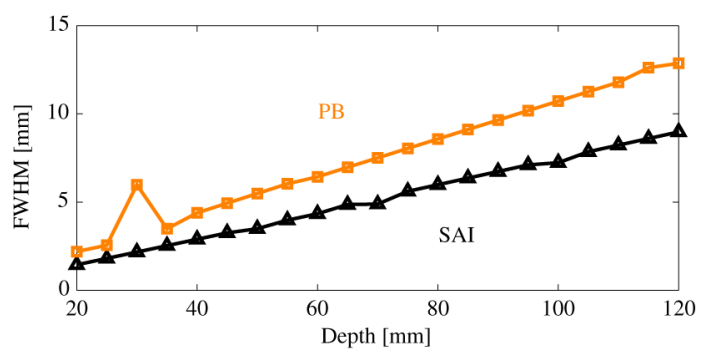

(a)

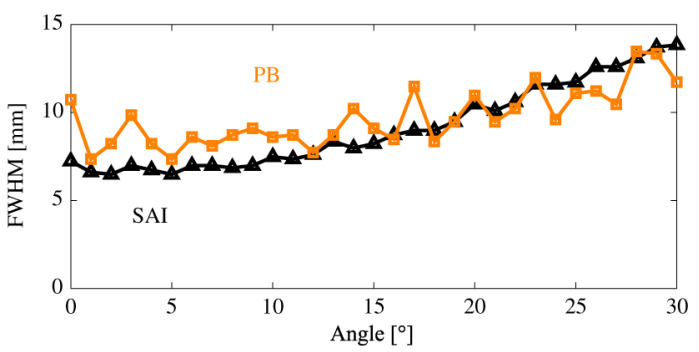

(b)

Fig. 9. The main-lobe size in (a) is seen as a function of depth with no tilt angle $\left[(\phi, \theta)=\left(0^{\circ}, 0^{\circ}\right)\right]$, and in (b) as a function of angle $[(\phi, \theta)=($ angle, angle)] at $100 \mathrm{~mm}$ of depth. For all depths in (a), the FWHM of the PSFs is seen to be slightly better for synthetic aperture imaging than for parallel beamforming. In (b) the FWHM performance improvement of synthetic aperture imaging is seen to be limited to angles up to about $10^{\circ}$ to $15^{\circ}$. At larger angles, the FWHM of parallel beamforming and synthetic aperture imaging are almost equal.

parallel beamforming transmit beams are illustrated with vertical dashed lines. Parallel beamforming performs better close to its transmit beams and worse between them. The synthetic aperture imaging sequence uses unfocused emissions, and no distinct performance oscillation is present.

\section{Measurements}

In this section, measurements made with synthetic aperture imaging and parallel beamforming are presented and compared.

\section{A. Measurement Setup}

All measurements are carried out using the 1024 channel experimental ultrasound scanner synthetic aperture real-time ultrasound system (SARUS) [41] and a $32 \times 32$ element phased array probe made by Vermon S.A. (Tours, France) shown in Fig. 11. The measured and simulated RF-data are beamformed using the Beam Formation Toolbox 3 [42]. For the measurements, two phantoms are used. The first phantom is a line-spread phantom consisting of a thin copper wire submerged in a water tank. The copper wire is mounted on the $x-y-z$ positioning system AIMS III made by Onda Corp. (Sunnyvale, CA) and can be placed precisely at any given depth. The second phantom is a cyst phantom, model 571 from Danish Phantom Design (Frederikssund, Denmark). The speed of sound $c_{\mathrm{pht}}$ and attenuation $\alpha$ of the cyst phantom are listed in Table I. The cysts are made of water-filled pipes orientated horizontally and with an angle of $45^{\circ}$ in the $x$-y-plane with respect to the scan plane. Two sizes of cysts are present in the phantom, cysts with a diameter of $8 \mathrm{~mm}$, spaced $20 \mathrm{~mm}$ apart, and cysts with a diameter of $4 \mathrm{~mm}$, spaced $10 \mathrm{~mm}$ apart. The contrast-to-noise ratio (CNR) is measured on the 8-mm cysts. The cysts are only present in the center of the phantom. The region without cysts contains only tissue-mimicking material and is used for estimating the SNR for both imaging techniques.

\section{B. Line Spread Function}

The line spread function (LSF) is measured using the described wire phantom. The wire runs parallel to the transducer surface at depths of 20,40,60, and $80 \mathrm{~mm}$. In Fig. 12, 2-D B-modes of the wire at $40 \mathrm{~mm}$ and $80 \mathrm{~mm}$ depths are shown. The B-mode images are sliced perpendicular to the wire direction. They have a dynamic range of $60 \mathrm{~dB}$, over which isocurves at $-40 \mathrm{~dB},-20 \mathrm{~dB}$, and $-6 \mathrm{~dB}$ are marked. The images in Figs. 12(a) and 12(b) are made with parallel beamforming and in Figs. 12(c) and $12(\mathrm{~d})$ with synthetic aperture imaging. The wire is placed a little off-center, resulting in slightly asymmetric LSFs. Comparing the LSFs of synthetic aperture imaging

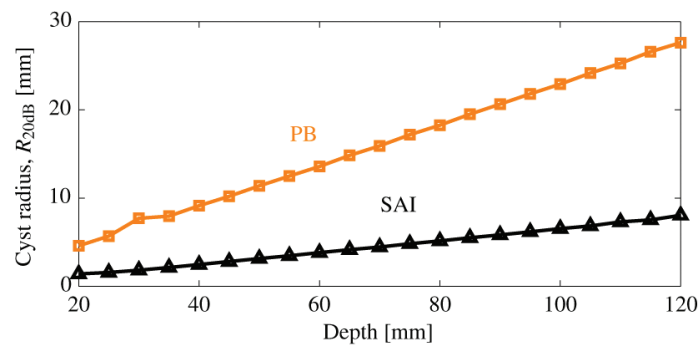

(a)

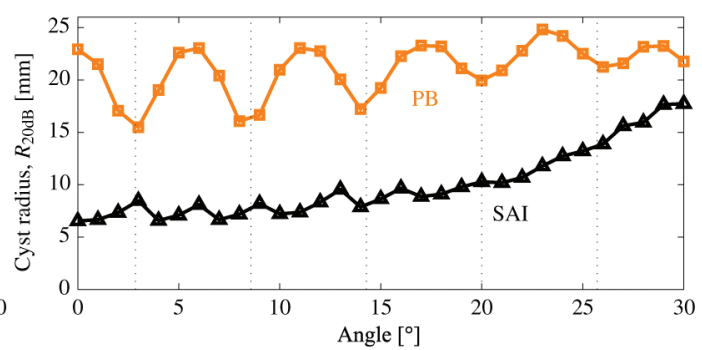

(b)

Fig. 10. The cystic resolution sampled at $20 \mathrm{~dB}$ intensity difference is in (a) seen as a function of depth with no tilt angle $\left[(\phi, \theta)=\left(0^{\circ}, 0^{\circ}\right)\right]$, and in (b) as a function of angle $[(\phi, \theta)=($ angle, angle) $]$ at $100 \mathrm{~mm}$ of depth. In (b), the angle of the parallel beamforming transmit beams are marked with vertical dashed lines. The smaller the cyst radius is, the better. The cystic resolution of parallel beamforming is seen to be improved near the transmit beams. 


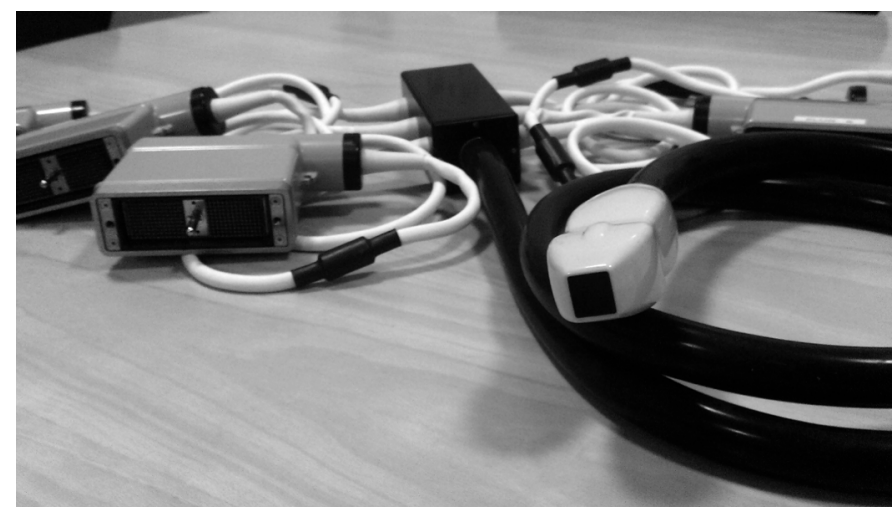

Fig. 11. The $32 \times 32$ element phased array ultrasound probe used for the measurements and modeled in the simulations.

and parallel beamforming, the -6 - $\mathrm{dB}$ contour widths differ $0.4 \mathrm{~mm}(16 \%)$ at $40 \mathrm{~mm}$ of depth and $1.0 \mathrm{~mm}(20 \%)$ at $80 \mathrm{~mm}$, in favor of synthetic aperture imaging. The FWHM of the LSF is shown in Fig. 12. The side-lobe levels are clearly lower for synthetic aperture imaging than for parallel beamforming, both at $-20 \mathrm{~dB}$ and at $-40 \mathrm{~dB}$. The received energy at the ultrasound probe surface is lower for deeper wire placements in the water tank. This results in a relative increase in the noise floor originating from electronic noise within the experimental ultrasound scanner. The noise floor is seen to be higher for parallel beamforming in Fig. 12(b) than for synthetic aperture imaging in Fig. 12(d). The noise appears at the center of the B-mode image, because the noise is in phase in the raw data on all receive channels and only sums up coherently during the delay-and-sum beamforming of points close to the center line pointing straight down.

In Fig. 13, the FWHM measured on the wire phantom at depths of 20,40,60, and $80 \mathrm{~mm}$ are shown. The FWHM increases linearly and the difference between the two techniques becomes larger with depth. The measured LSFs at $20 \mathrm{~mm}$ depth have a FWHM of $1.1 \mathrm{~mm}$ for synthetic aperture imaging versus $1.3 \mathrm{~mm}$ for the parallel beamforming technique. At $60 \mathrm{~mm}$, the FWHM has increased to $3.1 \mathrm{~mm}$ for synthetic aperture imaging versus $3.9 \mathrm{~mm}$ for parallel beamforming.

From the measured LSF, the cystic resolution is calculated and shown in Fig. 14, where a large difference between the imaging performances of the two techniques is apparent. At $20 \mathrm{~mm}$ depth, synthetic aperture imaging needs a cyst with a radius of $1.1 \mathrm{~mm}$ and parallel beamforming $1.3 \mathrm{~mm}$ for the center of the cyst to be $20 \mathrm{~dB}$ darker than its surroundings. At $60 \mathrm{~mm}$ depth, the required cyst size has increased to $2.5 \mathrm{~mm}$ for synthetic aperture imaging versus $4.1 \mathrm{~mm}$ for parallel beamforming. Relatively, synthetic aperture imaging thereby improves the cystic resolution by $15 \%$ at $20 \mathrm{~mm}$ and $39 \%$ at $60 \mathrm{~mm}$.

In Fig. 15, a 2-D slice of the cyst phantom is shown. Fig. 15(a) is made with synthetic aperture imaging and Fig. 15(b) with parallel beamforming. The cysts are clearly more apparent when imaged with synthetic aperture imaging than with parallel beamforming.

\section{Contrast-to-Noise Ratio}

The imaging performance of the cysts is estimated using the CNR, which was calculated as

$$
\mathrm{CNR}=\frac{\mu_{\mathrm{s}}-\mu_{\mathrm{c}}}{\sqrt{\sigma_{\mathrm{s}}^{2}+\sigma_{\mathrm{c}}^{2}}}
$$

where $\mu_{\mathrm{c}}$ and $\mu_{\mathrm{s}}$ are the mean intensities of a cyst region and a speckle region. $\sigma_{\mathrm{c}}^{2}$ and $\sigma_{\mathrm{s}}^{2}$ are the variances of the cyst and speckle data. The CNR is calculated from spherical regions of the envelope-detected data before it is logcompressed. The cyst statistics is measured from a sphere with a radius of $6 \mathrm{~mm}$ located at the center of each cyst. The speckle statistics are estimated on the exact same spheres but on the tissue-mimicking phantom containing only random scatterers. In Fig. 16, the results of the CNR calculations from the 8-mm cysts are shown. The CNR is seen to be better for synthetic aperture imaging at all depths compared with parallel beamforming. When ignoring the first cyst, the CNR for both synthetic aperture imaging and parallel beamforming cysts are seen to decrease approximately linearly. The CNR of parallel beamforming decreases faster than the CNR for synthetic aperture imaging. This is as expected when compared with the linearly decreasing contrast performance of the LSF, shown in Fig. 14, and the simulated cystic resolution, shown in Fig. 10(a). The decreased CNR performance of the top cyst could be a result of excessive clutter close to the transducer.

\section{Signal-to-Noise Ratio}

The SNR can be used to estimate the penetration depth. The SNR is measured by scanning a tissue-mimicking phantom only containing randomly distributed scatterers. To estimate the signal and noise components, 12 frames were acquired. The signal component is estimated by averaging the 12 measurements. Subtracting the signal component from each frame produces 12 noise estimates. The SNR is estimated for each voxel in the IQ-beamformed volume by taking the ratio of the signal power and the noise power. The SNR is thereby estimated on a complex data set. The RMS-power of a complex signal when assuming a unit load is

$$
P_{\mathrm{RMS}}=V_{\mathrm{RMS}} \times I_{\mathrm{RMS}}^{*}=V_{\mathrm{RMS}}{ }^{2},
$$

where the asterisk denotes complex conjugation. Using (8), the SNR is calculated by

$$
\begin{gathered}
\operatorname{SNR}(\mathbf{x})=\frac{\left|\sqrt{\frac{1}{N} \sum_{n=1}^{N} E[s(\mathbf{x})]^{2}}\right|^{2}}{\left|\sqrt{\frac{1}{N} \sum_{n=1}^{N}\left(s_{n}(\mathbf{x})-E[s(\mathbf{x})]\right)^{2}}\right|^{2}} \\
=\frac{|E[s(\mathbf{x})]|^{2}}{\operatorname{var}[s(\mathbf{x})]},
\end{gathered}
$$


PB

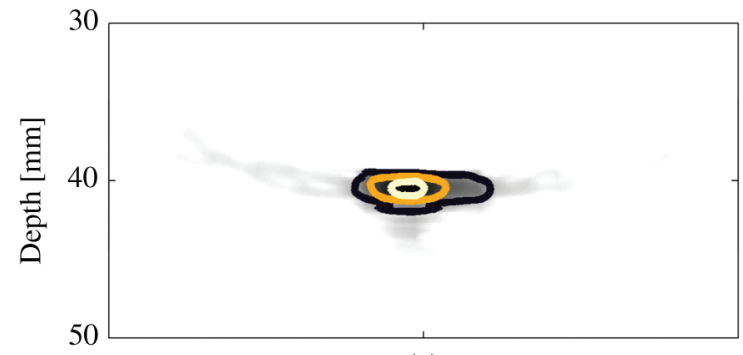

(a)

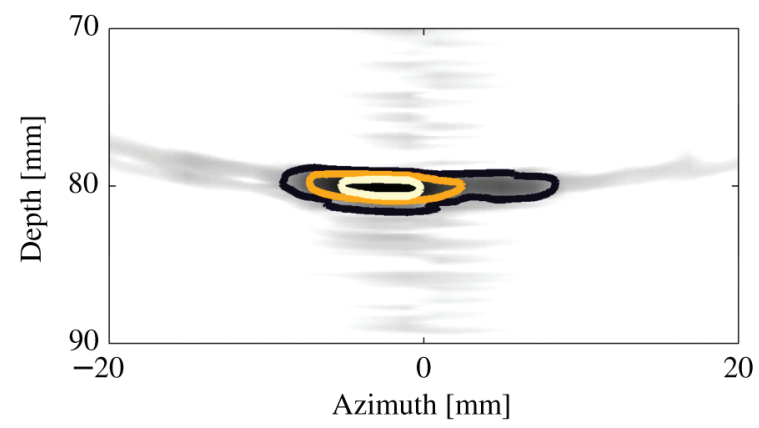

(b)
SAI

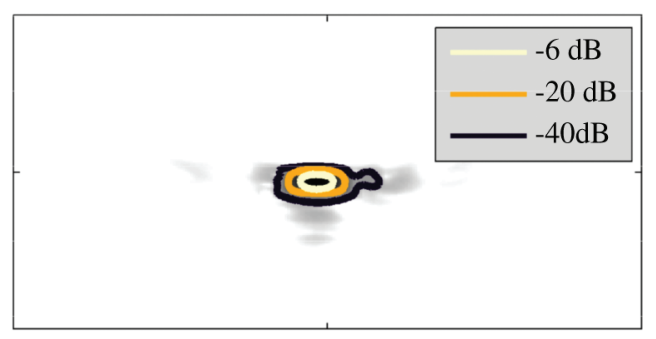

(c)

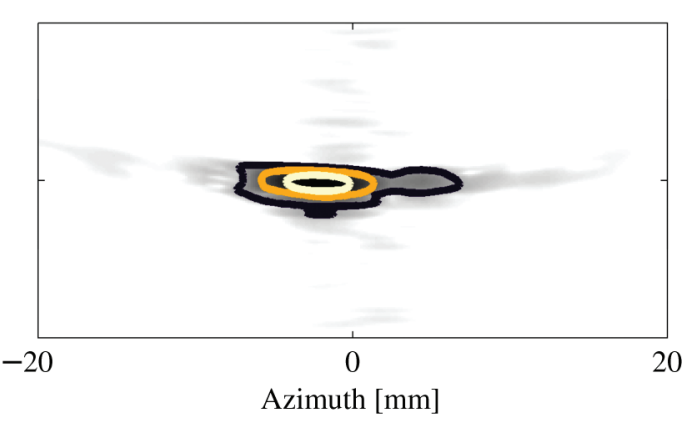

(d)

Fig. 12. The measured 3-D line spread function of parallel beamforming [(a), (b)] and synthetic aperture imaging [(c), (d)] sliced in a 2-D plane. The wire runs parallel to the transducer surface at depths of $40 \mathrm{~mm}$ and $80 \mathrm{~mm}$. The 2-D gray level images have a dynamic range of $60 \mathrm{~dB}$. The side-lobes of parallel beamforming are seen to be clearly larger than the side-lobes of synthetic aperture imaging.

where $\mathbf{x}=(x, y, z)^{\mathrm{T}}$ is the voxel coordinate, $N$ is the number of image frames, $E[\cdot]$ is the expectation operator, $\operatorname{var}[\cdot]$ is the variance operator, $s$ is the set of all IQ-beamformed frames, and $s_{n}$ is a single image frame with index $n$. The estimated SNR is calculated from stochastic data and a limited amount of data are available because of the depthdependent SNR. Therefore, averaging must be employed to reduce the variance of the estimates. The noisy estimates are low-pass filtered with a 3-D FIR filter. The FIR filter is applied before scan line conversion and its cut-off frequency for the three dimensions is $1 / 6^{\circ} \times 1 / 6^{\circ} \times 0.15 /$ $\mathrm{mm}$. A single scan line is extracted and plotted in Fig. 17. The SNR of synthetic aperture imaging is seen to be higher than the SNR of parallel beamforming. The penetration depth at which the SNR crosses $0 \mathrm{~dB}$ is estimated by linear regression to be $88 \mathrm{~mm}$ for parallel beamforming and $109 \mathrm{~mm}$ for synthetic aperture imaging. In other

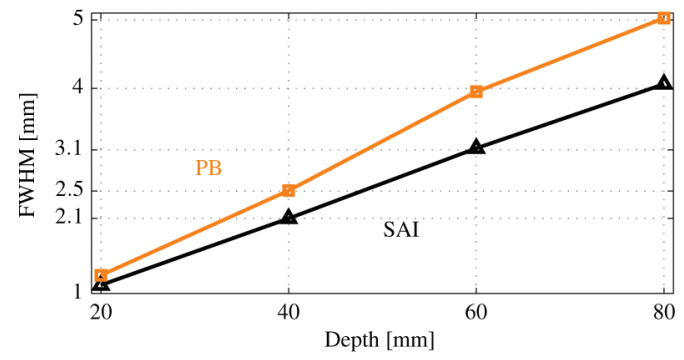

Fig. 13. The measured FWHM on the wire phantom shown as a function of wire depth. Synthetic aperture imaging has a smaller main-lobe size than parallel beamforming at all depths. The FWHM increases linearly with depth, as predicted by the simulations. words, synthetic aperture imaging increases the penetration depth by approximately $24 \%$ with the same amount of energy emitted.

\section{Discussion and Conclusions}

A comparison of real-time 3-D synthetic aperture imaging and parallel beamforming using only 256 active channels was made with both Field II simulations and measurements from the experimental ultrasound scanner SARUS.

Simulations were used to optimize both techniques before comparison. Simulations of the PSF at a large number of positions within the imaged volume showed that

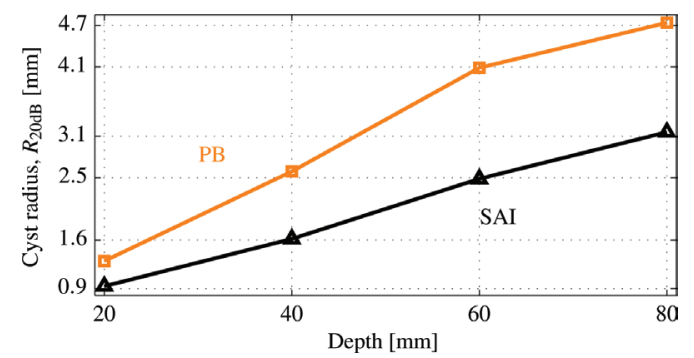

Fig. 14. The measured cystic resolution on the wire phantom shown as a function of wire depth. Synthetic aperture imaging is seen to achieve a better cystic resolution than parallel beamforming at all depths. The $R_{20 \mathrm{~dB}}$ values increase approximately linearly with depth, and they increase faster for parallel beamforming than for synthetic aperture imaging. This is in agreement with the simulation predictions. 
PB

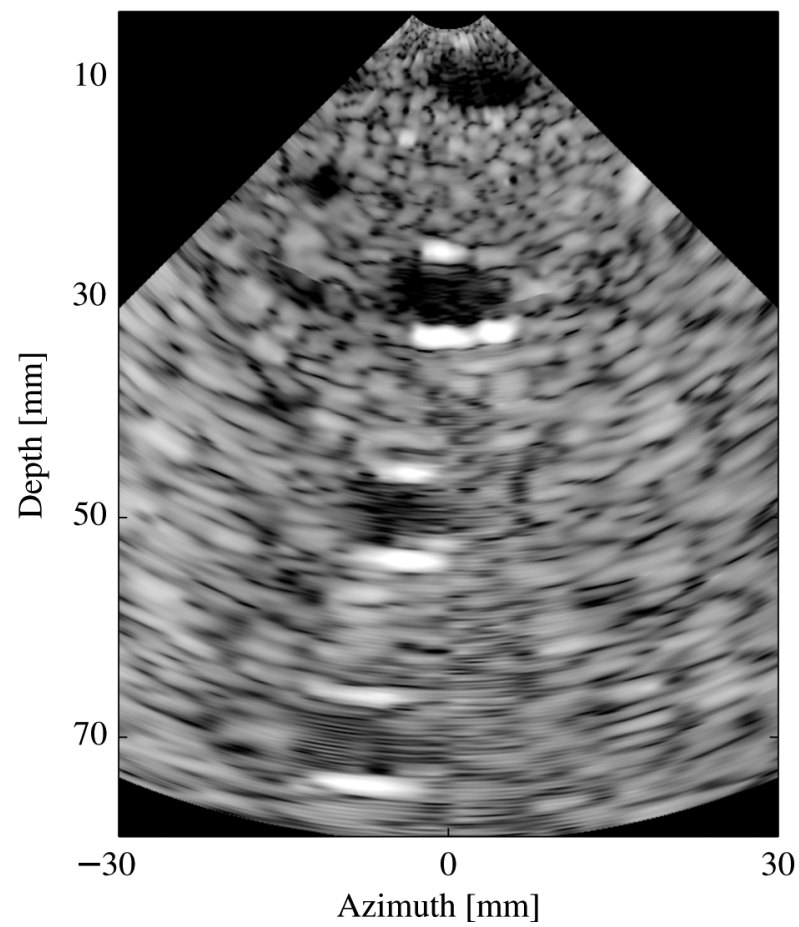

(a)

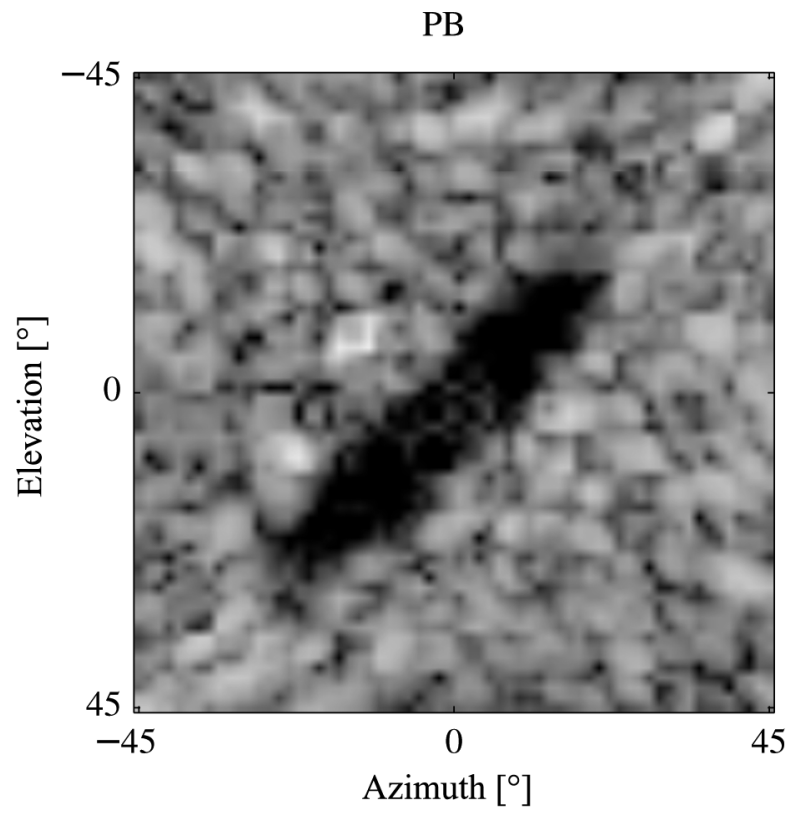

(c)
SAI

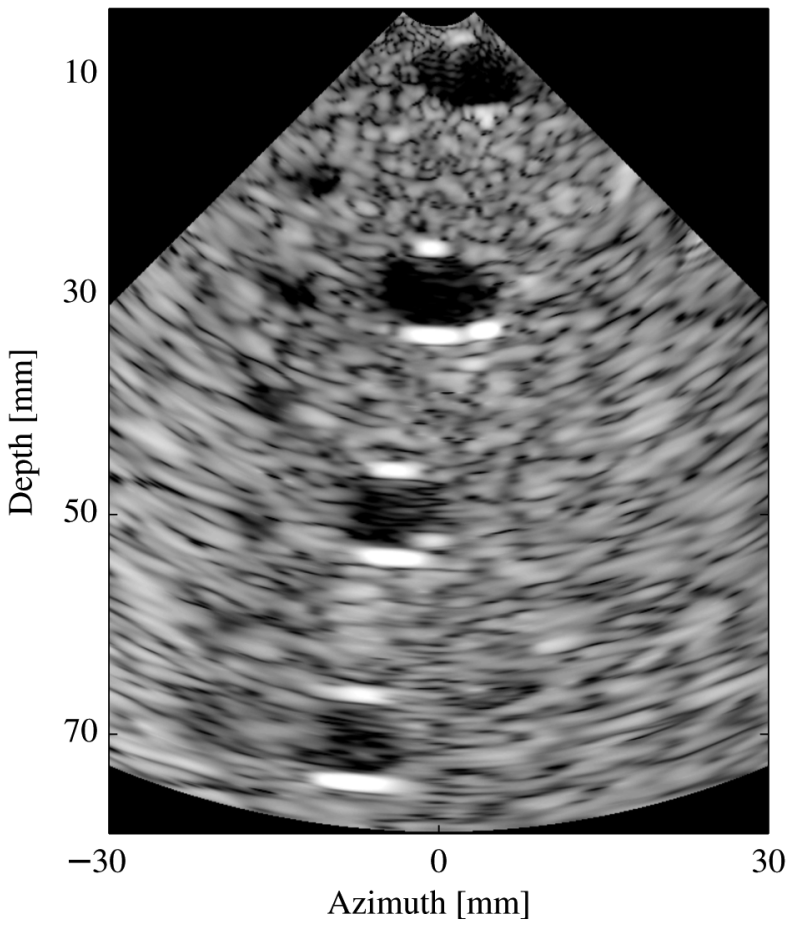

(b)

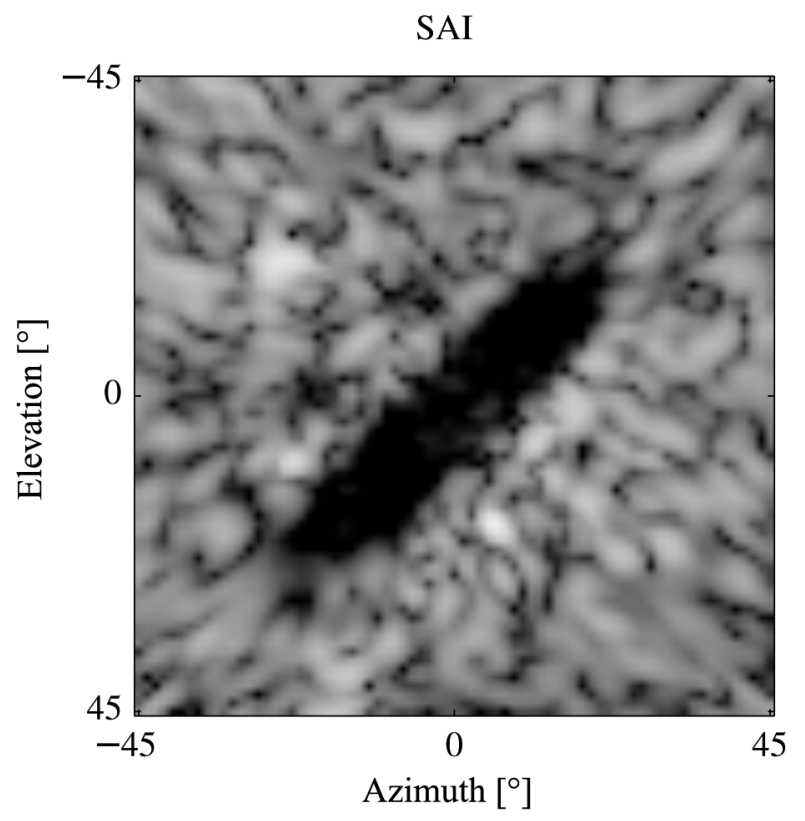

(d)

Fig. 15. Parallel beamforming [(a) and (c)] and synthetic aperture imaging [(b) and (d)] of anechoic cysts embedded in a tissue-mimicking phantom. The dynamic range is $40 \mathrm{~dB}$. The large cysts have a diameter of $8 \mathrm{~mm}$ and the small cysts $4 \mathrm{~mm}$. (a) and (b) are vertical scan planes and (c) and (d) are C-scans with a constant distance to the array center. The cysts are water-filled pipes aligned $45^{\circ}$ to the vertical scan plane.

synthetic aperture imaging improved the detail resolution at small steering angles compared with parallel beamforming. With larger steering angles, the detail resolution performance of the two techniques was similar. The contrast resolution was significantly improved by synthetic aperture imaging at all positions in the entire imaged volume.

From measurement, the imaging quality was investigated by use of wire and cyst phantoms. The wire phantom measurements were carried out at a low steering angle and showed the same tendency as the simulations. Synthetic aperture imaging increased both the resolution and the contrast. The FWHM was improved by $20 \%$ at $80 \mathrm{~mm}$ depth. The cyst phantom measurement confirmed that synthetic aperture imaging increases the contrast at all depths, compared with parallel beamforming. The CNR was improved by $22 \%$ at $70 \mathrm{~mm}$ depth. Measurements on 


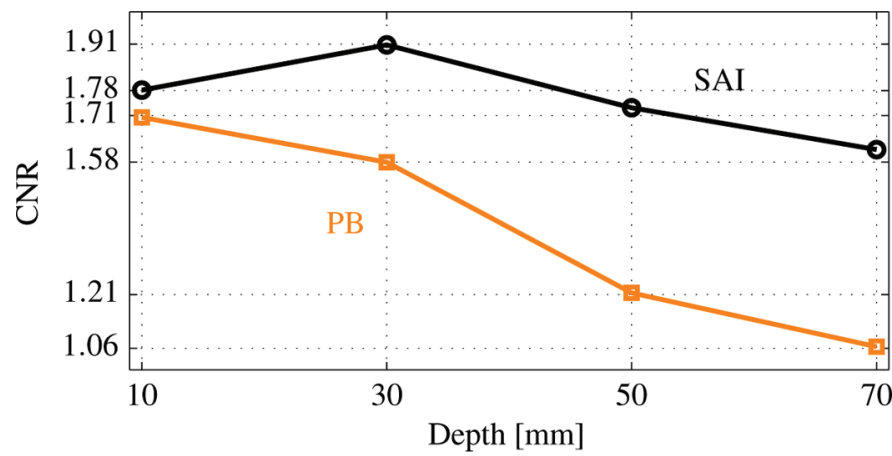

Fig. 16. The estimated contrast-to-noise ratios of the 8-mm cysts are shown. When ignoring the cyst at $10 \mathrm{~mm}$, this shows the same tendency as in the simulations in Fig. 10(a), the contrast decreases linearly with depth and the contrast for parallel beamforming decreases faster than for synthetic aperture imaging. The cyst at $10 \mathrm{~mm}$ depth is an outlier. This is probably because of reverberations from the phantom-transducer interface.

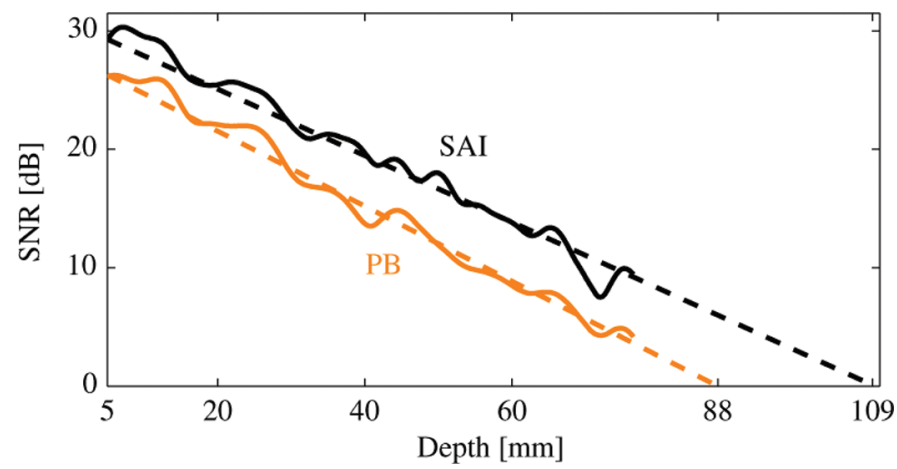

Fig. 17. Using synthetic aperture imaging increases the SNR at all depths. The penetration depth is estimated to $109 \mathrm{~mm}$ for synthetic aperture imaging and $88 \mathrm{~mm}$ for parallel beamforming.

a tissue-mimicking phantom indicated that the penetration depth is deeper for synthetic aperture imaging compared with parallel beamforming. Synthetic aperture had a higher SNR than parallel beamforming at all depths and the increased SNR resulted in a penetration depth increase of $24 \%$. The penetration depth did not reach the designed $15 \mathrm{~cm}$. This was likely due to the small transducer array surface area, limited by the restriction of 256 active channels. It is most likely that the low SNR of the experimental scanner used also limited the penetration depth.

It was shown that using synthetic aperture imaging on a channel-limited 3-D ultrasound system can achieve a high image quality at a low cost.

\section{REFERENCES}

[1] J. J. Wild, "The use of ultrasonic pulses for the measurement of biologic tissues and the detection of tissue density changes," Surgery, vol. 27, no. 2, pp. 183-188, 1950.

[2] C. B. Burckhardt, P.-A. Grandchamp, and H. Hoffmann, "An experimental $2 \mathrm{MHz}$ synthetic aperture sonar system intended for medical use," IEEE Trans. Sonics Ultrason., vol. 21, no. 1, pp. 1-6, Jan. 1974.
[3] S. Bennett, D. K. Peterson, D. Corl, and G. S. Kino, "A real-time synthetic aperture digital acoustic imaging system," in P. Alais and A. F. Metherell, Eds., Acoustic Imaging, vol. 10, 1982, pp. 669-692.

[4] J. J. Flaherty, K. R. Erikson, and V. M. Lund, "Synthetic aperture ultrasound imaging systems," U.S. Patent, 3548642, Dec. 22, 1970.

[5] J. T. Ylitalo and H. Ermert, "Ultrasound synthetic aperture imaging: Monostatic approach," IEEE Trans. Ultrason. Ferroelectr. Freq. Control, vol. 41, no. 3, pp. 333-339, 1994.

[6] M. Karaman, H. S. Bilge, and M. O'Donnell, "Adaptive multi-element synthetic aperture imaging with motion and phase aberation correction," IEEE Trans. Ultrason. Ferroelectr. Freq. Control, vol. 45, no. 4, pp. 1077-1087, 1998.

[7] M. Karaman and M. O'Donnell, "Subaperture processing for ultrasonic imaging," IEEE Trans. Ultrason. Ferroelectr. Freq. Control, vol. 45 , no. 1 , pp. $126-135,1998$.

[8] B. Delannoy, R. Torguet, C. Bruneel, E. Bridoux, J. M. Rouvaen, and H. Lasota, "Acoustical image reconstructions in parallel-processing analog electronic systems," J. Appl. Phys., vol. 50, no. 5, pp. 3153-3159, 1979

[9] D. P. Shattuck, M. D. Weinshenker, S. W. Smith, and O. T. von Ramm, "Explososcan: A parallel processing technique for high speed ultrasound imaging with linear phased arrays," J. Acoust. Soc. Am., vol. 75 , no. 4, pp. 1273-1282, 1984.

[10] M. F. Rasmussen, J. M. Hansen, G. Férin, R. Dufait, and J. A Jensen, "Preliminary comparison of 3D synthetic aperture imaging with Explososcan," in Proc. SPIE Medical Imaging, 2012, vol. 8320, art. no. $83200 \mathrm{~F}$

[11] M. F. Rasmussen, G. Férin, R. Dufait, and J. A. Jensen, "Comparison of 3D synthetic aperture imaging and explososcan using phantom measurements," in Proc. IEEE Ultrasonics Symp., 2012, pp. 113-116.

[12] L. J. Augustine, "High resolution multiline ultrasonic beamformer," U.S. Patent 4644795, Feb. 24, 1987.

[13] D.-L. D. Liu, J. C. Lazenby, Z. Banjanin, and B. A. McDermott, "System and method for reduction of parallel beamforming artifacts," U.S. Patent 6447452 , Sep. 10, 2002

[14] T. Hergum, T. Bjåstad, K. Kristoffersen, and H. Torp, "Parallel beamforming using synthetic transmit beams," IEEE Trans. Ultrason. Ferroelectr. Freq. Control, vol. 54, no. 2, pp. 271-280, Feb. 2007.

[15] O. T. von Ramm, S. W. Smith, and H. R. Pavy, "High speed ultrasound volumetric imaging system-Part II: Parallel processing and image display," IEEE Trans. Ultrason. Ferroelectr. Freq. Control, vol. 38, no. 2, pp. 109-115, 1991.

[16] L. F. Nock and G. E. Trahey, "Synthetic receive aperture imaging with phase correction for motion and for tissue inhomogenitiesPart I: Basic principles," IEEE Trans. Ultrason. Ferroelectr. Freq. Control, vol. 39, no. 2, pp. 489-495, 1992.

[17] K. L. Gammelmark and J. A. Jensen, "Multielement synthetic transmit aperture imaging using temporal encoding," IEEE Trans. Med. Imaging, vol. 22, no. 4, pp. 552-563, 2003.

[18] M. Karaman, P. C. Li, and M. O'Donnell, "Synthetic aperture imaging for small scale systems," IEEE Trans. Ultrason. Ferroelectr. Freq. Control, vol. 42, no. 3, pp. 429-442, 1995.

[19] C. H. Frazier and W. D. O'Brien, "Synthetic aperture techniques with a virtual source element," IEEE Trans. Ultrason. Ferroelectr. Freq. Control, vol. 45, no. 1, pp. 196-207, 1998.

[20] M. H. Bae and M. K. Jeong, "A study of synthetic-aperture imaging with virtual source elements in B-mode ultrasound imaging systems," IEEE Trans. Ultrason. Ferroelectr. Freq. Control, vol. 47, no. 6, pp. 1510-1519, 2000.

[21] G. R. Lockwood, J. R. Talman, and S. S. Brunke, "Real-time 3-D ultrasound imaging using sparse synthetic aperture beamforming," IEEE Trans. Ultrason. Ferroelectr. Freq. Control, vol. 45, no. 4, pp. 980-988, 1998.

[22] S. I. Nikolov and J. A. Jensen, "3D synthetic aperture imaging using a virtual source element in the elevation plane," in Proc. IEEE Ultrasonics Symp., 2000, vol. 2, pp. 1743-1747.

[23] J. A. Johnson, M. Karaman, and B. T. Khuri-Yakub, "Coherent-array imaging using phased subarrays. Part I: Basic principles," IEEE Trans. Ultrason. Ferroelectr. Freq. Control, vol. 52, no. 1, pp. 37-50, 2005.

[24] J. A. Johnson, O. Oralkan, S. Ergun, U. Demirci, M. Karaman, and B. T. Khuri-Yakub, "Coherent array imaging using phased subarrays. Part II: Simulations and experimental results," IEEE Trans. Ultrason. Ferroelectr. Freq. Control, vol. 52, no. 1, pp. 51-64, 2005. 
[25] J.-J. Kim and T.-K. Song "Real-time 3D imaging methods using 2D phased arrays based on synthetic focusing techniques," Ultrason. Imaging, vol. 30, no. 3, pp. 169-188, 2008.

[26] G. E. Trahey and L. F. Nock, "Synthetic receive aperture imaging with phase correction for motion and for tissue inhomogeneitiesPart II: Effects of and correction for motion," IEEE Trans. Ultrason. Ferroelectr. Freq. Control, vol. 39, no. 4, pp. 496-501, 1992.

[27] H. S. Bilge, M. Karaman, and M. O'Donnell, "Motion estimation using common spatial frequencies in synthetic aperture imaging," in Proc. IEEE Ultrasonics Symp., 1996, pp. 1551-1554.

[28] K. L. Gammelmark and J. A. Jensen, "Duplex synthetic aperture imaging with tissue motion compensation," in Proc. IEEE Ultrasonics Symp., 2003, pp. 1569-1573.

[29] K.-Y. Ho, Y.-H. Chen, C.-Z. Zhan, and A.-Y. Wu, "VLSI implementation of real-time motion compensated beamforming in synthetic transmit aperture imaging," in IEEE Int. Symp. Circuits and Systems, 2013, pp. 1893-1896.

[30] K. L. Gammelmark and J. A. Jensen, "2-D tissue motion compensation of synthetic transmit aperture images," IEEE Trans. Ultrason. Ferroelectr. Freq. Control, vol. 61, no. 4, pp. 594-610, Apr. 2014.

[31] M. Bae, H. Lee, S. B. Park, R. Yoon, M. H. Jeong, D. G. Kim, M. Jeong, and Y. Kim, "A new ultrasonic synthetic aperture tissue harmonic imaging system," in Proc. IEEE Ultrasonics Symp., 2008, pp. $1258-1261$.

[32] J. H. Rasmussen, M. C. Hemmsen, S. S. Madsen, P. M. Hansen, M. B. Nielsen, and J. A. Jensen, "Preliminary study of synthetic aperture tissue harmonic imaging on in-vivo data," in Proc. SPIE Medical Imaging, 2013, vol. 8675, art. no. 867512 .

[33] D. Vilkomerson, J. Greenleaf, and V. Dutt, "Towards a resolution metric for medical ultrasound imaging," in Proc. IEEE Ultrasonics Symp., 1995, pp. 1405-1410.

[34] K. Ranganathan and W. F. Walker, "Cystic resolution: A performance metric for ultrasound imaging systems," IEEE Trans. Ultrason. Ferroelectr. Freq. Control, vol. 54, no. 4, pp. 782-792, 2007.

[35] S. W. Smith, H. G. Pavy, and O. T. von Ramm, "High speed ultrasound volumetric imaging system - Part I: Transducer design and beam steering," IEEE Trans. Ultrason. Ferroelectr. Freq. Control, vol. 38, no. 2, pp. 100-108, 1991.

[36] M. Karaman, I. O. Wygant, O. Oralkan, and B. T. Khuri-Yakub, "Minimally redundant 2-D array designs for 3-D medical ultrasound imaging," IEEE Trans. Med. Imaging, vol. 28, no. 7, pp. 1051-1061, Jul. 2009.

[37] B. D. Steinberg, Principles of Aperture and Array System Design. New York, NY: Wiley, 1976.

[38] R. T. Hoctor and S. A. Kassam, "The unifying role of the coarray in aperture synthesis for coherent and incoherent imaging," Proc. IEEE, vol. 78 , no. 4, pp. 735-752, 1990.

[39] J. A. Jensen and N. B. Svendsen, "Calculation of pressure fields from arbitrarily shaped, apodized, and excited ultrasound transducers," IEEE Trans. Ultrason. Ferroelectr. Freq. Control, vol. 39, no. 2, pp. 262-267, 1992.

[40] J. A. Jensen, "Field: A program for simulating ultrasound systems," Med. Biol. Eng. Comput., vol. 34, suppl. 1, pt. 1, pp. 351-353, 1996.
[41] J. A. Jensen, H. Holten-Lund, R. T. Nilsson, M. Hansen, U. D. Larsen, R. P. Domsten, B. G. Tomov, M. B. Stuart, S. I. Nikolov, M. J. Pihl, Y. Du, J. H. Rasmussen, and M. F. Rasmussen, "SARUS: A synthetic aperture real-time ultrasound system," IEEE Trans. Ultrason. Ferroelectr. Freq. Control, vol. 60, no. 9, pp. 1838-1852, 2013.

[42] J. M. Hansen, M. C. Hemmsen, and J. A. Jensen, "An object-oriented multi-threaded software beamformation toolbox," in Proc. SPIE Medical Imaging, Mar. 2011, vol. 7968, art. no. 79680Y.

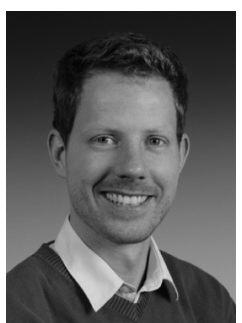

Morten F. Rasmussen was born in 1981. He received a B.Sc. degree and an M.Sc. degree in electrical engineering from the Technical University of Denmark (DTU), in 2008 and 2011, respectively. In 2007, he spent ten months at the Technische Universität Berlin, Berlin, Germany, working on the BeeSat satellite. He is currently pursuing his Ph.D. degree in electrical and biomedical engineering at the Center for Fast Ultrasound Imaging at DTU. The topics of his Ph.D. thesis are 3-D ultrasound imaging and synthetic aperture imaging techniques.

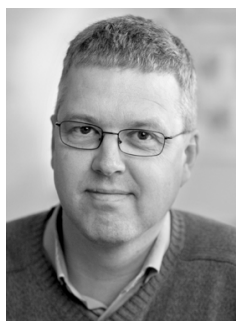

Jørgen Arendt Jensen earned his Master of Science degree in electrical engineering in 1985 and the Ph.D. degree in 1989, both from the Technical University of Denmark. He received the Dr.Techn. degree from the university in 1996 . He has published more than 250 journal and conference papers on signal processing and medical ultrasound and the book Estimation of Blood Velocities Using Ultrasound, published by Cambridge University Press in 1996. He is also developer of the Field II simulation program. He has been a visiting scientist at Duke University, Stanford University, and the University of Illinois at Urbana-Champaign. He is currently full professor of Biomedical Signal Processing at the Technical University of Denmark at the Department of Electrical Engineering and head of the Center for Fast Ultrasound Imaging. He has given courses on blood velocity estimation at both Duke University and the University of Illinois and teaches biomedical signal processing and medical imaging at the Technical University of Denmark. He has given several short courses on simulation, synthetic aperture imaging, and flow estimation at international scientific conferences. He has received several awards for his research and is an IEEE Fellow. His research is centered on simulation of ultrasound imaging, synthetic aperture imaging, vector blood flow estimation, and construction of ultrasound research systems. 